\title{
Numerical Investigation of the Flow Field in Confined Impinging Jets of Non Newtonian Fluids
}

\author{
A. Cavadas ${ }^{\mathrm{a}}$, F.T. Pinho, ${ }^{\mathrm{a}, \mathrm{b}}$ and J.M. Campos ${ }^{\mathrm{c}}$ \\ ${ }^{a}$ CEFT, DEMEGI, Faculdade de Engenharia, Universidade do Porto Rua Roberto Frias, 4200-465 Porto, Portugal \\ ${ }^{b}$ Universidade do Minho, Largo do Paço, 4704-553 Braga, Portugal \\ ${ }^{c}$ CEFT, DEQ, Faculdade de Engenharia, Universidade do Porto, Rua Dr. Roberto Frias, 4200-465 Porto, Portugal
}

\begin{abstract}
An extensive numerical investigation was carried out to assess the influences of shear-thinning intensity and Reynolds number on the steady flow of power law fluids within an impinging jet cell, and in particular, on the pressure loss and the size and strength of the recirculation region formed along the sloping surfaces of the cell. Some comparisons with experiment are reported. The size and strength of this recirculation increases with Reynolds number and threedimensional effects due to the finite aspect ratio of the geometry are reported. The end walls are shown to eliminate the recirculation in their vicinity and create a spiralling motion inside the recirculation. Shear-thinning enhances these effects. There is also a linear dependence between the pressure loss coefficient and the Reynolds number studied.
\end{abstract}

Keywords: Impinging jets, Non Newtonian fluids, shear-thinning fluids, Finite volume method.

PACS: 47.15.Uv; 47.50.-d; 47.11.Df; 47.15.Rq

\section{INTRODUCTION}

High velocity impinging jets are frequently used in industry to cool metals and process food and pharmaceutical products [1], because of the large increases in heat and mass transfer they promote near the impact surface. The jets may be confined or not and in this work a numerical investigation on the dynamics of isothermal laminar flows of impinging jets for non-Newtonian fluids is carried out. This follows a preliminary experimental programme reported elsewhere [2]. This jet issues from a rectangular duct where the flow becomes fully-developed and this study investigates the behaviour of power law fluids with power indices varying from 0.2 to 1 and inlet Reynolds number varying between 10 and 800 . At the conference results of predictions with viscoelastic models will also be presented.

\section{FLOW GEOMETRY, NUMERICAL METHOD AND FLUID MODEL}

The cell test section is schematically represented in Fig. 1(a). The test section is located downstream of a rectangular duct of height $(H)$ equal to $20 \mathrm{~mm}$ and width $(W)$ of $260 \mathrm{~mm}$. Its length is equal to $130 \mathrm{H}$ in order to fully-develop the flow prior to the impinging zone. The fully-developed flow exiting the duct impinges on a flat plate, confined by two slopping plane walls, each making an angle of $12^{\circ}$ relative to the plate. The two outgoing rectangular channels at the end of the cell have an height $h=4 \mathrm{~mm}$ high and a width (W) of $260 \mathrm{~mm}$.

The 3D numerical simulations were carried out with an in-house finite-volume method code, which has been described elsewhere [3-4]. Here, it suffices to say that all differencing and interpolating schemes were at least of $2^{\text {nd }}$ order. The computational grids were generated using patched blocks as is schematically in Fig 1(b). The mesh was non-uniform, with more control volumes (CV) in the cell zone for accurate predictions. The expansion factors used were carefully chosen to guarantee a smooth variation of CV size across the whole domain, in particular at the interfaces between the mesh-generating blocks. After tests on the adequacy of the computational domain and mesh size to ensure grid-independent results, the finest grid selected had a total number of $835200 \mathrm{CVs}$ with $30 \mathrm{CV}$ 's spanning the half-height of the channel. Symmetry relative to planes $x-y$ and $x-z$ was always considered based on experimental work [5], and preliminary numerical tests.

CP1027, The XV th International Congress on Rheology, The Society of Rheology $80^{\text {th }}$ Anmual Meeting

edited by A. Co, L. G. Leal, R. H. Colby and A. J. Giacomin

(02008 American Institute of Physics 978-0-7354-0549-3/08/\$23.00 
The fluids used in these simulations are described by a power law model and the predictions compared well with experimental results for Newtonian and non-Newtonian fluids. Fig. 1(c) compares the measured vertical velocity profile for a $0.2 \%$ by weight aqueous solution of xanthan gum at position $Y / H=0.8$ and $Z / W=0.45$ with the corresponding prediction for a Carreau model fluid matched to the shear viscosity of the polymer solution. There is good agreement between the two sets of data with a maximum difference of $10 \%$ even though the xanthan gum solution is viscoelastic. Consequently, it was decided to perform an extensive set of numerical calculations to investigate shear-thinning effects o the flow dynamics using a power law model.

In this work, the Kozicki Reynolds number is used $\left(R e^{*}\right)[6]$, which is based on the mean inlet velocity, U, the hydraulic diameter of the rectangular channel and an average viscosity.

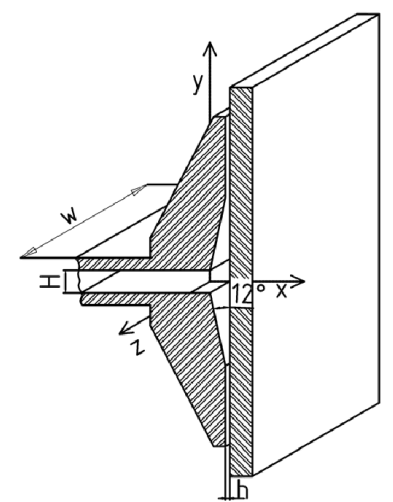

(a)

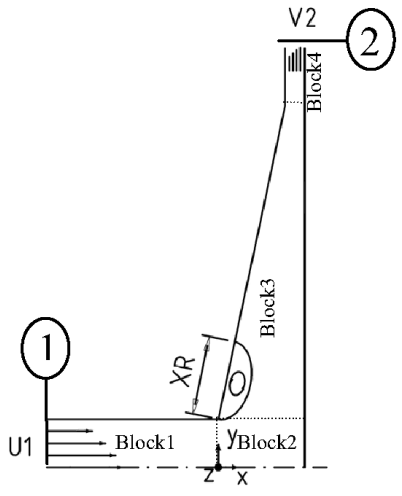

(b)

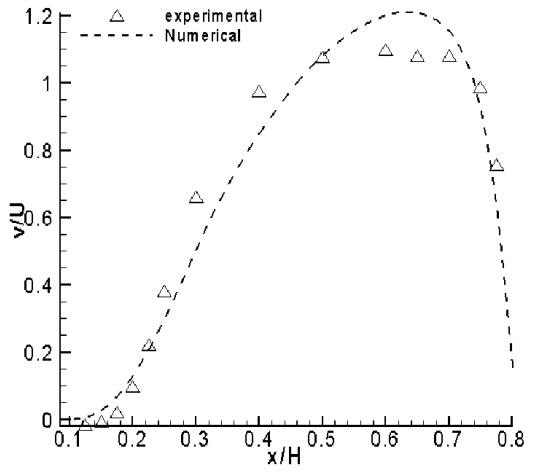

(c)

FIGURE 1. (a) Sketch of the impinging jet test section; (b) Blocks used to generate grid (c) Comparison between experimental data for $0.2 \%$ xanthan gum in water and numerical predictions for a fitted Carreau model at $Y / H=0.8, Z / W=0.45$.

\section{RECIRCULATION LENGTH, PRESSURE LOSS AND 3D EFFECT}

The spanwise variation of the recirculation bubble length $\left(X_{\mathrm{R}}\right)$ with $R e^{*}$ is shown in Fig. 2(a) for Newtonian fluids. $X_{\mathrm{R}}$ is constant over most of the span, with the side walls eliminating the separated flow region. The width of the central region of constant $X_{\mathrm{R}}$ decreases with $R e^{*}$ suggesting the strong coupling between $3 \mathrm{D}$ effects and inertia: for $R e^{*}=100$ the central region occupies $70 \%$ of the span, decreasing to $50 \%$ at $R e^{*}=800$. At the higher $R e^{*}$ it is noticeable that the largest $X_{\mathrm{R}}$ is not at the centre plane, but at the edge of the 3D affected region and this effect is actually enhanced by shear-thinning as is clearly show in Fig. 2(b) where all the profiles are for $R^{*}=200$.

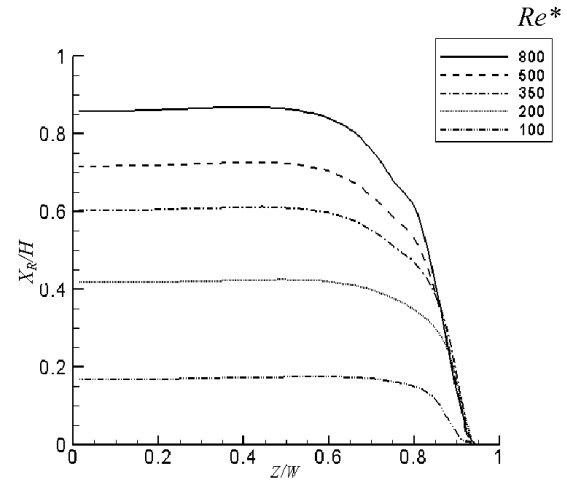

(a)

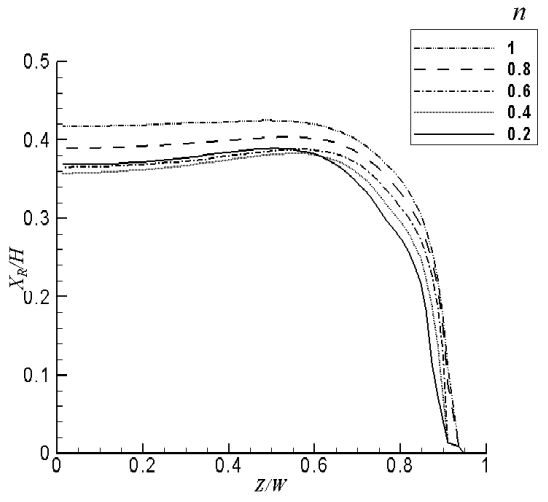

(b)

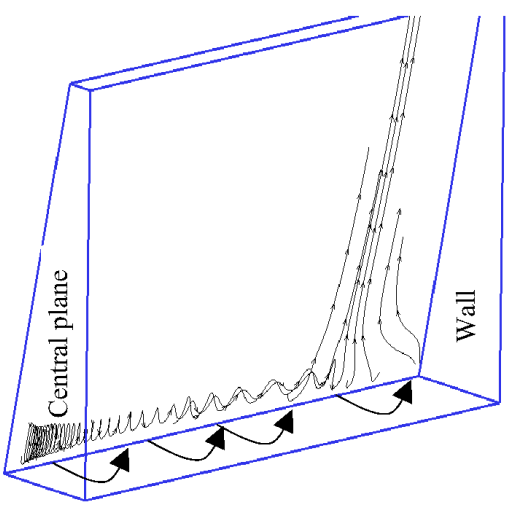

(c)

FIGURE 2. (a) Recirculation Length (XR) along the spanwise direction, $n=1$ versus $\mathrm{Re}^{*}(\mathrm{~b})$ Recirculation length $\left(\mathrm{X}_{\mathrm{R}}\right)$ along the spanwise direction for $\mathrm{Re}^{*}=200$ versus n; (b) Streamtraces in the recirculation zone for $\mathrm{Re}^{*}=200$; (c) Streamtraces in the recirculation zone for $\mathrm{Re}^{*}=200$;

Fig. 2(b) shows also that shear-thinning reduces $X_{\mathrm{R}}$ everywhere, except in the central region for $n=0.2$. The relative variation of $X_{\mathrm{R}}$ near the walls is also stronger than in the middle of the cell and shear-thinning enhances the appearance of the peak $X_{\mathrm{R}}$ at $Z / W \approx 0.6$, thus decreasing the width of the central region of constant. 
The three-dimensional flow inside the separated flow region consists of an helical motion along the spanwise direction from the centreplane towards the flat walls, as represented by the streaklines of Fig. 2(c). As the fluid in helical motion approaches the side wall it leaves the smaller recirculation zone creating a local jet seen in Fig. 3(a) as a local maximum. These three-dimensional effects are not numerical artifacts and were confirmed experimentally using flow visualization techniques (c.f. Fig. 3(a) and [5]).

The recirculation length at the centreplane is plotted in Fig. 3(b) as a function of $R e^{*}$ and shear-thinning intensity. At $\operatorname{Re}^{*}=800$, the behavior is essentially opposite to that found at $\operatorname{Re}^{*}=200$ with the smallest separation for the Newtonian fluid and the longest pertaining to the most shear-thinning fluid.

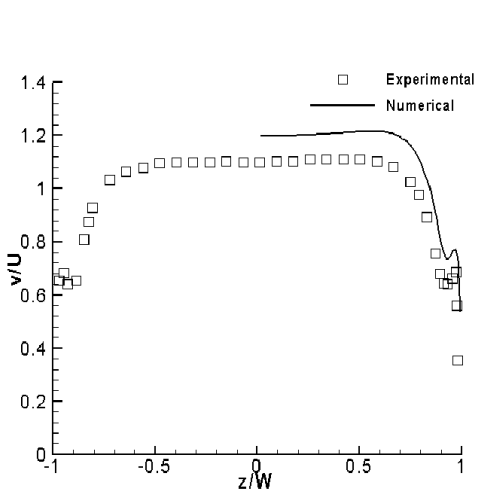

(a)

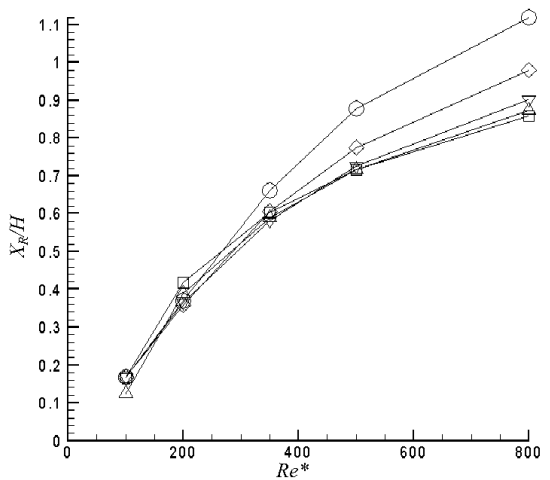

(b)

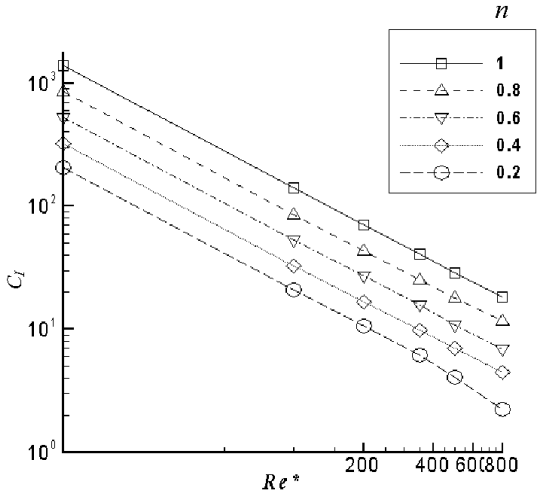

(c)

FIGURE 3. (a) Spanwise profiles of streamwise velocity at $X / H=0.525, Y / H=1.65$ with xantham gum at $\mathrm{Re}^{*}=200$; (b)Recirculation length versus $\mathrm{Re}^{*}$ for several power law indexes; (c) Predicted loss coefficient $\left(\mathrm{C}_{\mathrm{I}}\right)$ versus Reynolds number for different values of $n$.

The calculated pressure loss coefficient in the cell, $C_{\mathrm{I}}$, was determined from the variation of pressure along the geometry starting and ending in regions of fully-developed flow (planes 1 and 2 in Fig. 1b)). $C_{\mathrm{I}}$ is plotted in Fig. 3 (c) as a function of Kozicki Reynolds number and shear-thinning intensity in log-log coordinates. A linear dependence of $C_{\mathrm{I}}$ with $R e^{*}$ is seen for each value of $n$, especially for $\operatorname{Re}^{*}<400$ and $n \geq 0.6$. The plot also shows a reduction of $C_{\mathrm{I}}$ with shear-thinning when using this Reynolds number definition.

To summarize, the numerical calculations showed the main features of the flow within the cell and quantified in depth the variations of the recirculation length and of the pressure drop coefficient with $n$ and $R e^{*}$. $X_{\mathrm{R}}$ was seen to increase with $R e^{*}$ regardless of power law index, but shear-thinning made the behavior non-monotonic: at low $R e^{*}$ the largest values of $X_{\mathrm{R}}$ pertained to Newtonian fluids, whereas at large $R e^{*}$ the Newtonian fluids had the smallest recirculations. The numerical predictions were able to capture and understand better the $3 \mathrm{D}$ effects observed by flow visualization. The pressure loss coefficient was seen to vary in inverse proportion to the Reynolds number as is typical of flow dominated by viscous effects, in spite of the large Reynolds numbers involved, for all values of $n$.

\section{ACKNOWLEDGMENTS}

The authors acknowledge funding by CEFT and FCT and FEDER via Projects POCI/EQU/56342/2004, $\mathrm{PTDC} / \mathrm{EQU}-\mathrm{FTT} / 70727 / 2006$ and $\mathrm{PhD}$ grant BD/7091/2001.

\section{REFERENCES}

1. A. Sarkar, N. Nitin, M.V. Karwe and R.P. Singh, Journal of Food Science, Vol. 69, pp 113-122, (2004).

2. A.S. Cavadas, "Hydrodynamic of confined impinging jets, flow of Newtonian and non-Newtonian fluids" (in Portuguese), $\mathrm{Ph} . \mathrm{D}$. Thesis, Porto University, 2008.

3. P.J., Oliveira, F.T., Pinho, G.A., Pinto, J. Non-Newtonian Fluid Mech., 79, 1-43, (1998)

4. M.A. Alves, P.J., Oliveira, and F.T.Pinho, Int. J. Numer. Meth. Fluids, Vol. 47, pp 665-679, (2003)

5. A. S. Cavadas, J.B.L.M. Campos and F. T. Pinho, "Flow filed in a liquid impinging jet confined by slopping plane walls" 13th International symposium on applications of laser techniques to fluid mechanics, Lisbon, 26-29 June 2006

6. W. Kozicki, C.H. Chou, and C. Tiu, Chem. Eng. Sci., Vol. 21, pp 665-679, (1966). 